\title{
PANEL DISCUSSION ON REDSHIFT PROBLEMS
}

The "redshift controversy" can be said to have originated from studies of pairs and compact groups. There are two potential problems involving the measured redshifts of galaxies in interacting systems: 1) the question of the physical association of galaxies in pairs and groups with discordant $\left(\Delta \mathrm{V}_{0} \geq 10^{3} \mathrm{kms}^{-1}\right.$ redshift and 2) the possible quantization of redshifts in samples of pairs regarded (on the basis of redshift difference) as physical. The emphasis in the panel discussion was on the latter problem.

Each panel member gave an opening statement followed by discussion. In lieu of a direct transcript of the panel the contributors have summarized their presentations and impressions in the following contributions. - Eds.

\section{W. G. Tifft}

At the very heart of this IAU meeting on the dynamics of pairs and systems of galaxies there is an underlying assumption that the redshift is a simple Doppler shift which we may use to measure motions and build dynamical models. For 20 years now I have been working to test this underlying assumption using studies of 1) correlations of redshift with intrinsic properties of galaxies, 2) tests of continuity, commonly referred to as 'redshift quantization', and most recently 3 ) tests for rapid variability. A remarkably consistent overall picture has emerged which is not critically tied to any single aspect of the work. The result, simply put, is that the redshift has properties which are not consistent with a standard velocity interpretation. This result is based upon a multitude of samples including a considerable amount of high quality $21 \mathrm{~cm}$ data.

Studies in the 1970-1975 period led to discovery of various redshift-magnitude and redshiftmorphology correlations. These correlations are as unexplained today as they were then. In 1973 the first hints of quantization were presented at IAU Symposium 58. By 1975 the quantization concept was central in my thinking since it provided a very critical and clear test of conventional wisdom. Formal predictions were published in a series of three papers in the Astrophysical Journal in 1976. This original prediction of a basic redshift periodicity of $72.5 \mathrm{~km} \mathrm{~s}^{-1}$ was based primarily upon data in the Coma cluster and a review of internal dynamics of galaxies.

The 1976 predictions were not based significantly on any data on pairs of galaxies, but studies of pairs provided the first critical test of quantization. There now exist three independent accurate samples of pairs: 1) $21 \mathrm{~cm}$ pairs, beginning with the sample by $\mathrm{S}$ Peterson 
and evolving through several iterations into what we now refer to as the revised radio sample, 2) Optical studies of close Karachentsev pairs observed at Steward Observatory, and 3) A subsample of pairs observed optically by L Schweizer in the southern hemisphere. The most recent summary of work on pairs appeared in the Jan 1989 ApJ.

The picture for pairs is now quite sophisticated. The $\Delta \mathrm{V}$ distribution has at least three peaks $\left(24,72,145 \mathrm{~km} \mathrm{~s}^{-1}\right)$ and four minima $\left(0,48,108,180 \mathrm{~km} \mathrm{~s}^{-1}\right)$. My one illustration for this brief review shows the composite of the three available samples to illustrate the current situation. Each sample alone provides a significant test of the 1976 predictions. Among the newest results, discussed in the January 1989 ApJ, is the conclusion that identical redshifts do not occur in close proximity. This 'zero deviation' concept is at distinct variance with conventional dynamics, and stands alone as a fundamental redshift test. Several new tests based upon this 'exclusion' concept have been developed and are discussed in the January ApJ paper.

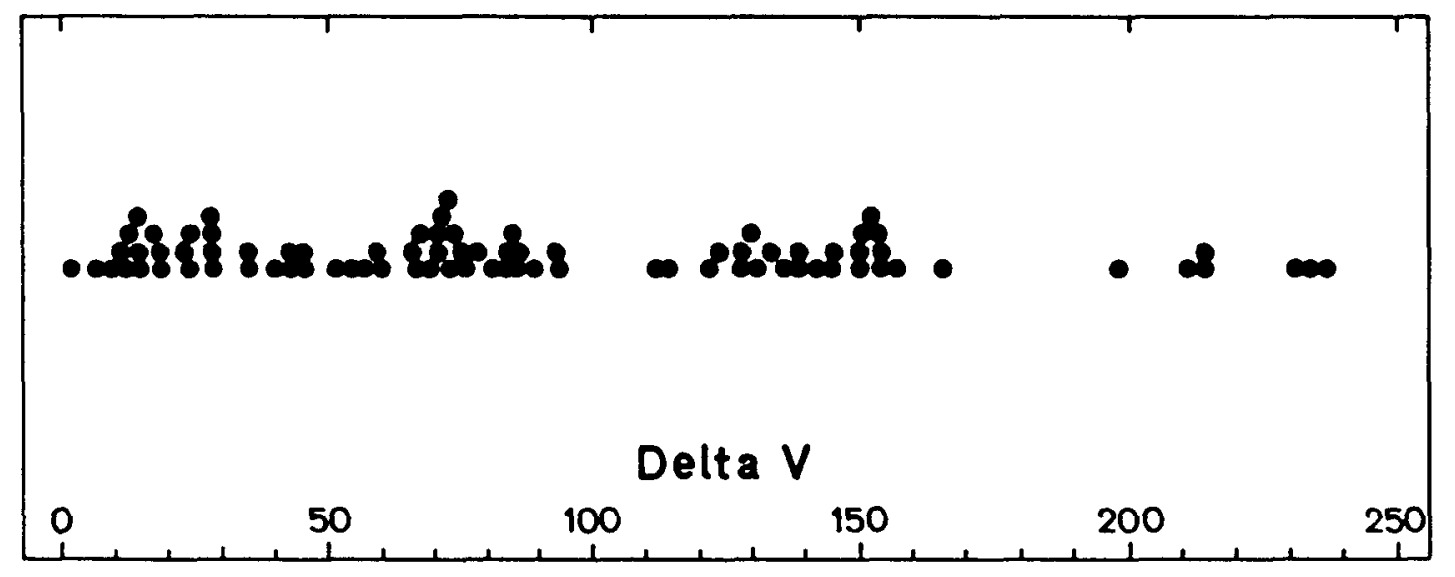

Figure 1. Cumulative differential redshift distribution of 84 points from the three best data sets, the revised radio sample (18), the Schweizer sample (25) and the high-weight Karachentsev pairs (41).

Recently Newman, Haynes and Terzian (ApJ 1989) have questioned the statistical framework used to test for quantization. They point out that clumping is commonly seen in small samples and is rarely significant. They do not account, however, for the fact that the particular pattern involved was specifically predicted in advance and not determined from the samples tested. Furthermore, multiple independent samples are involved. An interesting debate is also developing over the statistical approach used (Schneider and Sharp in this 
panel discussion, Sharp (1990), Cocke and Tifft (1990 ApJ in process) and Newman, Haynes and Terzian). No one has yet explained away the simple fact that predicted patterns have reoccured.

The quantization test was extended to the studies of groups of galaxies in 1983, again successfully. Important work showing quantization in groups has also been done by Arp and Sulentic. Bill Napier gave a paper at the Venice Symposium in 1987 which independently confirmed many of these studies. Steve Schneider of this panel was involved in work at Cornell which also found the $72 \mathrm{~km} \mathrm{~s}^{-1}$ peak in redshift differentials. They hope to explain this peak by selection (a study I strongly encourage). There are multiple peaks and valleys, however, including the very important hole at zero which they must also address. Groups are perhaps the most complex place to look for quantization effects. They are much more complex than simple isolated pairs. An attempt to predict what one might expect to find for triplets is contained in the January ApJ paper.

Clusters of galaxies also have an important role in redshift studies. The original quantization period came primarily from the Coma cluster analysis in 1976. Recent work using 21 cm data has confirmed the periodicity and was summarized in the Venice Symposium review in 1987. Guthrie and Napier have recently studied the Virgo cluster and find evidence for redshift periodicity.

A completely independent approach to redshift quantization has considered the redshift globally. In 1984 John Cocke and I demonstrated that redshifts of Fisher-Tully dwarf galaxies were globally quantized at $24.15=72.45 / 3 \mathrm{~km} \mathrm{~s}^{-1}$ after removal of the solar motion within the Galaxy. A second demonstration utilized the galaxies with wide $21 \mathrm{~cm}$ profiles which were periodic at $72.45 / 2 \mathrm{~km} \mathrm{~s}^{-1}$. In a very important paper (ApJ 345, 1989) Martin Croasdale has taken a new sample of galaxies from work by Giovanelli and associates and confirmed the global effect.

Quite recently John Cocke and I have extended the global quantization test to Lyman alpha forest lines in quasars. By combining simple theory, which suggests that the quantization interval should vary as $\mathrm{H}^{1 / 2}$, with conventional Friedman cosmology, it is possible to relate the quantization interval, $\mathrm{z}$, and $\mathrm{q}_{o}$. We find the expected quantization if $\mathrm{q}_{o}$ is close to $1 / 2$. This suggests to us that important aspects of conventional cosmology are intimately related to the quantization phenomenon. It is unlikely we will fully understand either without the other.

The newest, and perhaps the most stringent, test of the redshift concerns rapid time variability. Recent precision $21 \mathrm{~cm}$ data appears to indicate that redshifts are variable at 
the level of $1 \mathrm{~km} \mathrm{~s}^{-1} \mathrm{y}^{-1}$. The effect is intimately related to redshift quantization with the rate of change being a function of 'phase' within the periodicity. This phenomenon, along with an unexpected dependence of redshift on radius within galaxies, is the subject of Poster 49 at this colloquium. The first evidence for rapid variability was presented at the Venice Symposium in 1987. The first follow-up confirmation tests have now been made. By the time the new 300 foot radio telescope comes on line in the 1990s the predicted changes will have grown well outside of any reasonable uncertainty.

The main message of this brief introductory review is simply to point out that the failure of the redshift to pass muster as a simple Doppler shift is a broad one. The question does not hang on simply pairs or clusters or global periodicities, it cuts across many interlinked aspects which must be approached comprehensively. The redshift can very likely be 'cosmological'. It very likely can be a velocity, albeit constrained in some quantum framework. It seems very unlikely to me, however, that any approach to galaxies or cosmology which ignores the redshift question and proceeds on purely conventional dynamical grounds can be correct.

\section{S. E. Schneider}

This discussion involves the two most dangerous topics known to mankind: religion and statistics. For some reason, we have camps of 'believers' and 'non-believers' in discordant redshifts, and curiously the non-believers represent the 'orthodoxy.' With the black art of statistics being used as ammunition by both sides, we have a most explosive mixture! I have seen three attitudes at this meeting, covering the gamut of opinion about the significance of the whole topic of peculiar and discordant redshifts in groups of galaxies:

(1) There is no problem. The non-believers feel that if the statistics were properly handled, all of the discrepancies would fade away. It is indeed true that most of the claimed discrepancies depend on statistical arguments, and these arguments are rarely of a sufficiently clearcut character to allow a simple interpretation. Frequently, 'levels of confidence' of $95 \%$ or $99 \%$ are quoted for apparently discrepant data, but this amounts to only two or three statistical sigmas. I was taught not to believe anything less than five sigma, which if you think about it is an absurdly stringent requirement - something like a $99.9999 \%$ confidence level. But of course such a high limit is used because the actual size of sigma is almost never known. There are just too many free variables in astronomy that we can't control or don't know about. None of the discrepancies I am aware of have reached the five sigma level yet, although not all are readily amenable to statistical analysis. 
I would like to see the non-believers work more at finding those overlooked variables that would reduce the statistical significance of some of the claimed redshift discrepancies. And publish them! All too often quick explanations are offered up like bad excuses, without careful consideration. We have heard claims and counter-claims at this meeting about the significance of the large number of compact groups with a discordant-redshift member. It is now being suggested that the high number seen can be explained by simple foreground or background contamination, although it is by no means obvious to most of us why this should be so common. Careful interloper studies, accounting for large- scale structure along the line of sight to these compact groups are reportedly underway (Paul Hickson, this meeting), and I hope we'll see the results soon.

(2) There is a problem that cannot be explained within the framework of standard astronomy. At the opposite extreme, the believers find there are some phenomena so contradictory to expectation that no statistical argument is needed. Redshifts that only take on values at discrete intervals, nearby galaxies with luminous connections to quasars, groups of galaxies in which the faint members all have higher redshifts than the brightest member - these phenomena are all plainly at odds with the standard model, and defy explanation.

Here, though, I fear we delve into a murky area of statistics. Patterns found in old data are not amenable to standard statistical analyses. Newman, Haynes, and Terzian (1989) have shown, for example, that periodicities can be found in random data, and they may incorrectly appear to be statistically significant. They demonstrate that too much freedom in the possible parameterization of the periodicity invalidates the standard statistical tests. Thus, statistical significance cannot be established unless there are prior constraints on the total dimensions of the possible 'hypothesis space.' Unfortunately, most of the discordant redshift data falls in the category of being found in current data sets. I would like to see the believers define the limits of their hypothesis space. For example, in an alignment between a quasar jet and an unusual galaxy at entirely different redshifts, what degree of alignment is adequate? with the inner or outer jet? at what separation? should there be one on each side? should all jets point at similar type galaxies? Until these and other possibilities are clearly laid out in advance of the observations, the significance of putative connections between objects with widely different redshift cannot be established. Most important of all, testable predictions must be made for future observations.

(3) There is a problem, but it can be explained within the framework of standard astronomy. The agnostics just don't know whether they don't believe. Probably most of us fall in this category. We would never claim that the standard model is infallible, and we are 
intrigued by the strange discrepancies being reported.

Bill Tifft's 'redshift quantization' is one of the most intriguing claims. I think his binary galaxy data passes most of the statistical objections raised above (at least as well as most papers I read in the Ap.J.), and it is even getting beyond the three sigma level by some measures. I am worried by the evolution I see in the hypotheses being made about the important redshifts to consider, but his findings provide the the possibility of making testable predictions about binary galaxy orbits. My own analysis suggests that the phenomenon he discovered in binaries is probably caused by selection effects, but I suspect it may still provide a key to understanding the nature and cosmological origins of binary galaxy orbits (see my contribution elsewhere in this volume). Other phenomena like group members with discordant redshifts and redshift asymmetries in groups may also have explanations in the realm of 'interesting' physics rather than 'new' physics, like the suggestions by Valtonen and Byrd that dynamical ejections may be occurring. I hope we will see more effort put into these areas. The rewards are potentially large since the most exciting discoveries in science often come from resolving the discrepant observations with the standard model.

\section{G. Burbidge}

Over more than 20 years the observational evidence has grown that QSOs with large redshifts are to be found close to galaxies with small redshifts more frequently than expected by chance. The earlier evidence has been summarized by Burbidge (1979) and by Arp in his book (Arp 1987).

The most recent and most extensive study is that by Burbidge, Hewitt, Narlikar and das Gupta (in press in ApJ Supplements). They have shown that among the QSOs now known there are nearly 600 lying within $10^{\prime}$ of about 500 galaxies. This includes the small number of small redshift QSOs (about 40) known to be close to galaxies at the same redshift. The bulk of the remainder lie close to bright galaxies with very different (smaller) redshifts. Statistical analysis of this large sample shows that QSOs are preferentially found near galaxies, regardless of their redshifts. When looked at quantitatively, gravitational microlensing cannot explain these results. The conclusion is that a significant part of the redshifts of QSOs cannot be attributed to the expansion of the universe.

Most recently there has been further analysis of the distribution of redshifts of QSOs, and periodicity in the redshift distribution has been reconfirmed. This periodicity may be related to the periodicity found in analyzing the redshifts of faint galaxies. 


\section{N. A. Sharp}

It is true that there are puzzling aspects to the distribution of redshifts, both in the issue of discordant redshift companions (which I have also studied), and in the area of debate here, which is the claim of a strong periodicity. I can only address this claim as it relates to double or binary galaxies, but, as it happens, this is one area where the conventionally quite inexplicable period seems to appear most convincingly.

Note that we have no physical theory for periodicity (just some attempts at a mathematical representation, which is not the same thing at all). Although one may yet appear, this lack accounts for at least some of the opposition to the very idea of non-cosmological, non-Doppler redshifts. In addition, remember that even if there is a compelling periodicity in the data, we are still not forced to conclude that it is present in the underlying physical systems. One might also puzzle over a quantization which appears in a radial measurement of redshift, which might seem to require that our Galaxy be somehow specially placed.

However, my main focus here is that the data are not as compelling as is suggested.

In the face of the highly periodic figures commonly shown for double galaxy systems, I am sometimes tempted to shout 'Foul !', due to the number of caveats and 'yes, but ...' remarks one wants to make.

Among the early work, claims were made that a strong period shows up in only the radio data collected by Peterson in his thesis work (Peterson, 1979), and that since radio-derived redshifts are more accurate than optical values, it must be more convincing. However, the data actually entering into that claim were not purely radio data, and some radio data were excluded. Although the reasons for exclusion were stated in the original paper, they are not repeated in other forums, and any and all a posteriori selection is suspect when we consider such a radical claim. The purely radio data make a much less compelling picture (Sharp, 1990).

A very accurate set of optical data was recently collected by Schweizer (1987), and held to reveal a strong periodicity. The original Schweizer data do not show a period at all (Sharp, 1990), and especially not at anything even remotely approaching the significance that a sample of this size and accuracy should reveal. But again the argument goes that we must exclude part of the data set in order to bring out the real physical effect. However, in the Schweizer sample, it is only the closest pairs which really begin to reveal the effect, whereas the previous 'best bet' samples were radio data, which are well-known to have much wider separations than optically-selected pairs (Sharp, 1990).

So, perhaps we should first ask, why is it that contradictory selections must be applied to 
different samples, if the effect is physical ?

On a different tack, comparisons between different authors' data for the same physical pairs suggest that the internal error estimates are rather low (not uncommon in astronomy), and that some samples should not show the period they seem to show, because the redshifts just aren't accurate enough (Sharp, 1990). Croasdale (1989) shows the other side of this same coin, in work on the 'global' quantization rather than on binaries, suggesting that the data errors had to be much larger than estimated in order to degrade the period to the level found. Although error analysis is complex and disputatious, again we find reasons to be cautious.

Another point was introduced by Newman et al. (1989), and followed up in a number of private conversations and correspondences which I hope will one day be restated for publication. The (very controversial) claim is that the ' $\mathrm{I} / \mathrm{O}$ ' statistic used to show that the period is highly significant is not, in fact, a properly designed statistic with a known and rigorously derived error distribution. Technically, the I/O value does not have 'statistical power', and is not discriminatory. This point has been, and no doubt will continue to be, strenuously argued. However, it is significant that the non-parametric Kolmogorov-Smirnov statistic (Sharp, 1984) showed no significant deviation from a classical model of double galaxy orbital motions, until it was reduced to a form equivalent to that of the $I / O$ statistic. In retrospect, having looked again at the derivation of the error distribution for the KS test, I believe that the folding I used did considerable violence to the calculation, and probably vitiates the statistical conclusion. In passing, I would also like to emphasise that my 1984 paper is in no way an independent proof of periodicity: quite to the contrary, I found no discrepancy with the complex dynamical simulation method until after I used both the same data sets and an equivalent test of significance.

I take a skeptical stance: an effect of this importance should not be ignored, but I have serious doubts about the selection, presentation, and accuracy of the data. I return a Scottish verdict of 'Not proven'. 